\title{
Micro-Flow Imaging: Flow Microscopy Applied to Sub-visible Particulate Analysis in Protein Formulations
}

\author{
Deepak K. Sharma, ${ }^{1,2}$ Dave King, ${ }^{1}$ Peter Oma, ${ }^{1}$ and Clark Merchant ${ }^{1}$
}

Received 21 September 2009; accepted 12 May 2010; published online 2 June 2010

\begin{abstract}
The need to monitor, measure, and control sub-visible proteinaceous particulates in biopharmaceutical formulations has been emphasized in recent publications and commentaries. Some of these particulates can be highly transparent, fragile, and unstable. In addition, for much of the size range of concern, no practical measurement method with adequate sensitivity and repeatability has been available. A complication in measuring protein particulates in many formulations is the simultaneous presence of other particle types such as silicone micro-droplets, air bubbles, and extrinsic contaminants. The need has therefore been identified for new analytical methods which can accurately measure and characterize sub-visible particulates in formulations. Micro-flow imaging has been shown to provide high sensitivity in detecting and imaging transparent protein particles and a unique capability to independently analyze such populations even when other particle types are present.
\end{abstract}

KEY WORDS: light obscuration; micro-flow imaging; particle sizing; protein aggregation; protein formulation.

\section{INTRODUCTION}

Recent commentaries have stressed a need for characterizing sub-visible particle populations in protein drug formulations (1). Particles of primary concern are protein aggregates in the size range from approximately 0.1 to $50 \mu \mathrm{m}$. For particles larger than $1 \mu \mathrm{m}$, often present in relatively low concentrations, individual particle rather than composite population measurement techniques are applicable. A complication in measuring protein particulates in many formulations is the simultaneous presence of other particle types such as silicone oil micro-droplets, air bubbles, and extrinsic contaminants. A measurement method which could isolate different particle sub-populations would thus be highly desirable. Light obscuration (LO) and membrane microscopy are the standard methods for measuring extrinsic particles $>10 \mu \mathrm{m}$. However, these techniques underestimate populations of smaller transparent particles $(2,3)$, are highly material dependent, and cannot distinguish sub-populations. Therefore, these technologies are not ideal for this application, and no other practical measurement method having adequate sensitivity and accuracy has been available. These challenges have led to an interest in alternative technologies which are less dependent on particle properties and can provide additional information on particle parameters.

\footnotetext{
${ }^{1}$ Brightwell Technologies Inc, 115 Terence Matthews Crescent, Ottawa, Ontario, Canada K2M 2B2.

${ }^{2}$ To whom correspondence should be addressed. (e-mail: dsharma@ brightwelltech.com)
}

Micro-flow imaging (MFI) has been shown to provide high sensitivity in detecting and imaging transparent particles and a unique capability to differentiate sub-populations of particles having different morphologies using image filtering $(4,5)$. This paper reviews work carried out by Brightwell and its collaborators in evaluating, optimizing, and applying MFI technology to measure sub-visible particles in protein formulations.

\section{MFI TECHNOLOGY}

MFI is a flow microscopy technology, where bright field images are captured in successive frames as a continuous sample stream passes through a flow cell centered in the fieldof-view of a custom magnification system having a wellcharacterized and extended depth-of-field. The instrument configuration is shown in Fig. 1. A standard bench-top MFI configuration uses a simple fluidics system, where sample fluid is drawn either directly from a pipette tip or larger container through the flow cell using a peristaltic pump. The combination of system magnification and flow-cell depth determines the accuracy of concentration measurement. Concentration and parameter measurements are absolute but may be re-verified using particle standards. Typical sample volumes range from $<0.25$ to tens of milliliters. Frame images displayed during operation provide immediate visual feedback on the nature of the particle population in the sample. The system software extracts particle images by using a sensitive threshold to identify pixel groups which define each particle. Successive frames, each containing many 


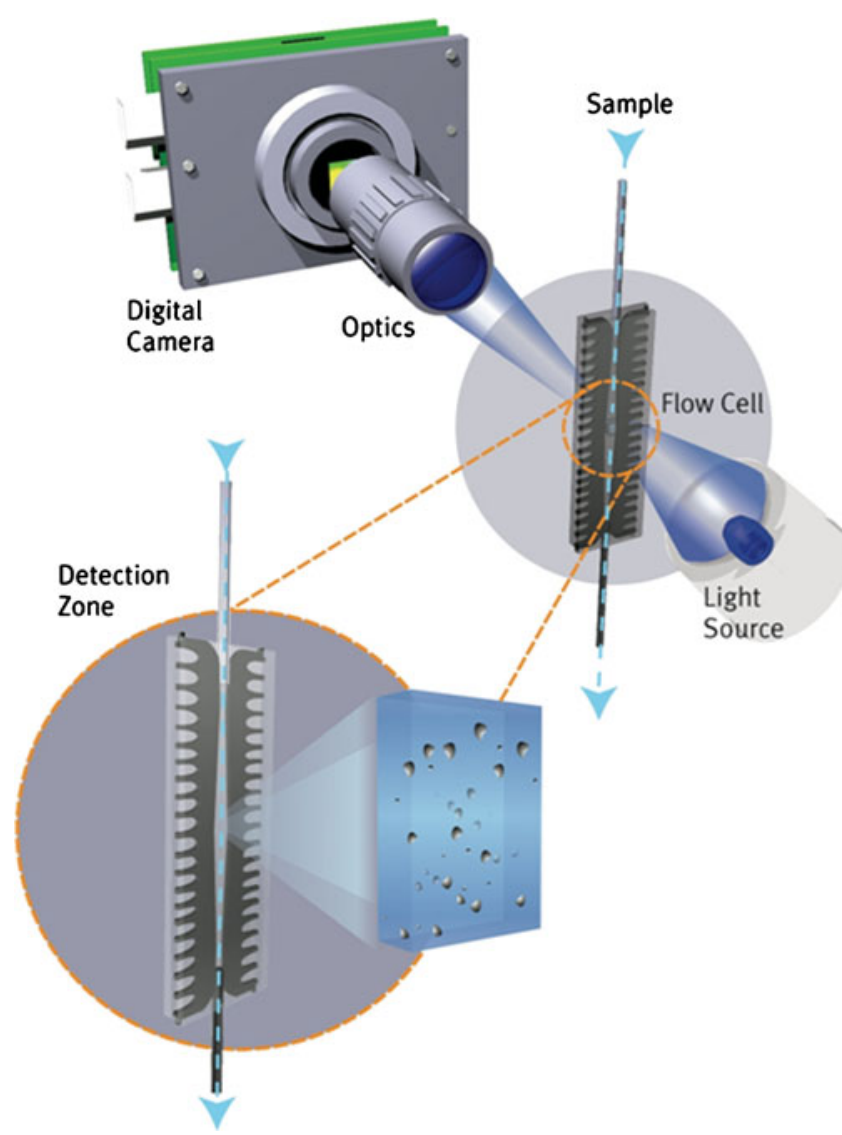

Fig. 1. Micro-flow imaging instrument configuration

particle images, are analyzed in real time. Maximum instrument sensitivity for detecting near-transparent particles is achieved by automatically optimizing threshold values, using low-noise electronics, implementing noise reduction algorithms, and compensating for all possible non-uniformities in spatial and pulse-to-pulse illumination. Ten-bit grayscale resolution is used to improve threshold accuracy.

Images are analyzed to compile a database containing count, size, concentration, as well as a range of shape and image contrast parameters. This database is interrogated by the computer's application software to produce parameter distributions using histograms and scatter plots. The software supports image filtering by calculating a trial filter based on user selected representative particles and then interacting with the user to optimize this filter to extract similar particles from the total population. This feature allows particle subpopulations to be isolated and independently analyzed. Particle images are available for verification, further investigation, and analysis. Once a successful assay has been developed and validated, the resulting protocol, including run parameters, software filters, and report formats, can be saved for future use.

Direct imaging particle measurement technologies have a number of advantages over indirect obscuration or scattering-based measurements. They do not rely on a correlation between particle size and the magnitude of a scattered or obscured optical signal as calibrated using polystyrene reference beads. Provided the contrast in the particle image is sufficient for the pixels to be resolved by the system threshold, the particle will be detected and measured. No calibration by the user is required. The particle images captured by the system also provide qualitative and quantitative information about the target particle population. Qualification studies based on National Institute of Standards and Technology-traceable polystyrene beads have shown that the technology can meet high standards for sizing, concentration accuracy, and repeatability (3). Representative performance parameters for MFI instrument configurations optimized for applications related to sub-visible particle analysis in protein formulation development are shown in Table I.

Work to be described in the sections below includes comparison of MFI with USP < 788> methods, measurement studies on a variety of typical protein formulations, morphological software filter development, assessment of consistency and repeatability of the technology, and optimization of MFI technology for this application.

\section{PROTEIN PARTICLES AND FLOW MICROSCOPY}

\section{Comparison of MFI and USP $<788>$ Methods}

An early study compared the performance of the MFI technology with USP $<788>$ method (manual microscopy and light obscuration) for an engineered parenteral formulation containing proteinaceous particles (3). For particle sizes in the $<788>$ size range $(\geq 10 \mu \mathrm{m})$, the measured concentrations for this sample type differed by one or more orders of magnitude and became larger as the particle size increased. Similar results have been reported using different proteinbased formulations in other pharmaceutical laboratories $(5,6)$. The significantly lower count by manual microscopy is most likely due to a combination of factors such as break-up of

Table I. Typical Micro-Flow Imaging Performance Parameters

\begin{tabular}{|c|c|c|}
\hline Parameters & DPA-4100 model & DPA-4200 model \\
\hline Size range $(\mu \mathrm{m})$ & 2 to 300 & 1 to 70 \\
\hline Concentration range w/o dilution (counts/mL; $2.5 \mu \mathrm{m}$ objects) & 175,000 & 900,000 \\
\hline Analysis rate $(\mu \mathrm{L} / \mathrm{min})$ & 193 & 150 \\
\hline Sampling efficiency (\%) & \multicolumn{2}{|c|}{85} \\
\hline Sizing accuracy $(\% ; 5 \mu \mathrm{m}$ particles $)$ & \multicolumn{2}{|c|}{ \pm 5} \\
\hline Concentration accuracy ( $\% ; 5 \mu \mathrm{m}$ particles $)$ & \multicolumn{2}{|c|}{ \pm 10} \\
\hline Measurement outcome & \multicolumn{2}{|c|}{ Particle size, concentration (counts/mL), and shape/morphology } \\
\hline Intended use/suitable for & \multicolumn{2}{|c|}{ Research, development, and quality control } \\
\hline
\end{tabular}


aggregates during sample preparation protocol and operator interpretation. The origin of the differences in results between flow microscopy and light obscuration has not been fully investigated. One of the contributing factors is likely to be the minimal refractive index difference between protein particles and the carrier fluid, producing obscuration signals which are smaller than those obtained from calibration beads of equivalent size. This weaker interaction of protein particles with the illumination source is directly observed by visual inspection of recorded images from MFI which show clear differences in intensity (transparency) between typical protein aggregates and the polystyrene reference beads (Table II).

To investigate the effects of refractive index matching between target particles and their carrier fluids, suspensions of low refractive index glass beads were prepared in aqueous carrier fluids whose refractive index was increased with the addition of ethylene glycol. The effect of increasing refractive index can be seen in Figs. 2a (LO), and 2b (MFI). These figures show size and concentration measurements for a population of $4.80 \mu \mathrm{m}$ low refractive index glass beads (refractive index 1.43 to 1.46 at $589 \mathrm{~nm}$ ) suspended in water (refractive index $\sim 1.33$ ) and in a solution of $40 \%(v / v)$ ethylene glycol in water (refractive index $~ 1.37$ ). Measurements are in agreement between the two techniques when the beads are suspended in water. However, significant undersizing is seen for the light obscuration measurement in the higher refractive index ethylene glycol mixture, while flow microscopy results are almost unaffected. The refractive index of various protein aggregates have been reported in the range of 1.33 to $1.40(7,8)$ versus 1.59 for polystyrene bead standards. While less susceptible to these effects than light obscuration, results obtained by flow microscopy can also be affected by particle transparency. For highly transparent particles found in some formulations, the contrast of some or all of the pixels in the particle image may not be sufficient to trigger the system threshold. These particles will then be under-sized, seen as fragmented images or missed entirely. Post-analysis of saved images can be used to determine whether particles are being under-sized or fragmented. With proper choice of instrument configuration, these effects can be minimized for samples containing protein particles.
While refractive index matching of particle and carrier fluid may be an important component in the differences seen between measurement techniques, other factors could also contribute. These include:

- Differences in the optical absorption of proteins at different wavelengths (in this case the LO light source wavelength was $780 \mathrm{~nm}$ and the flow microscope light source was $470 \mathrm{~nm}$ )

- Impact of particle morphology on sizing

- Particle fragmentation at higher shear forces induced by higher flow rates in LO

- Undetected coincidence effects in LO

- Particle settling or fragmentation resulting from inadequate or excessive magnetic stirring in LO

\section{Application Studies on Protein Formulations}

To evaluate the suitability of MFI for characterizing subvisible particles in protein formulations, the technique has been applied to a variety of formulation and aggregate types which might be encountered in the development and manufacturing of bio-pharmaceuticals.

\section{Stress-Induced Aggregates}

Processes such as fermentation, purification, formulation, filling, shipment, and storage that can result in stress induced aggregation are always encountered during manufacturing of bio-pharmaceuticals (9-12). Stress-induced aggregate populations were produced by a freeze-thaw procedure in a protein formulation containing monoclonal IgG1 antibody $(1 \mathrm{mg} / \mathrm{mL})$ as the model protein in phosphate-buffered saline (PBS) buffer $(\mathrm{pH} 7.0 \pm 0.2)$. The count, size, and morphological characteristics of the protein particulates were analyzed using MFI (4). The protein particles or aggregates were observed to be highly heterogeneous in shape, ranging from small dense fibers to large ribbon-like aggregates. Figure 3 contains representative images of protein aggregates with a wide range of values for the morphological parameters such as circularity, aspect ratio and intensity.

Table II. Comparison between Protein Particles and Polystyrene Beads

\begin{tabular}{|c|c|c|c|c|}
\hline \multicolumn{5}{|c|}{ Polystyrene beads } \\
\hline Parameters & $\bullet$ & & & \\
\hline Size $(\mu \mathrm{m})$ & 10 & 20 & 30 & 50 \\
\hline Intensity mean $(0-1023)$ & 463.47 & 316.62 & 249.22 & 182.54 \\
\hline Circularity (0-1) & 0.93 & 0.92 & 0.94 & 0.94 \\
\hline \multicolumn{5}{|c|}{ Protein particles } \\
\hline Parameters & 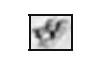 & 40 & & \\
\hline Size $(\mu \mathrm{m})$ & 10 & 20 & 30 & 50 \\
\hline Intensity mean $(0-1023)$ & 746.87 & 730.6 & 687.73 & 632.29 \\
\hline Circularity (0-1) & 0.64 & 0.48 & 0.39 & 0.26 \\
\hline
\end{tabular}


a

Light Obscuration

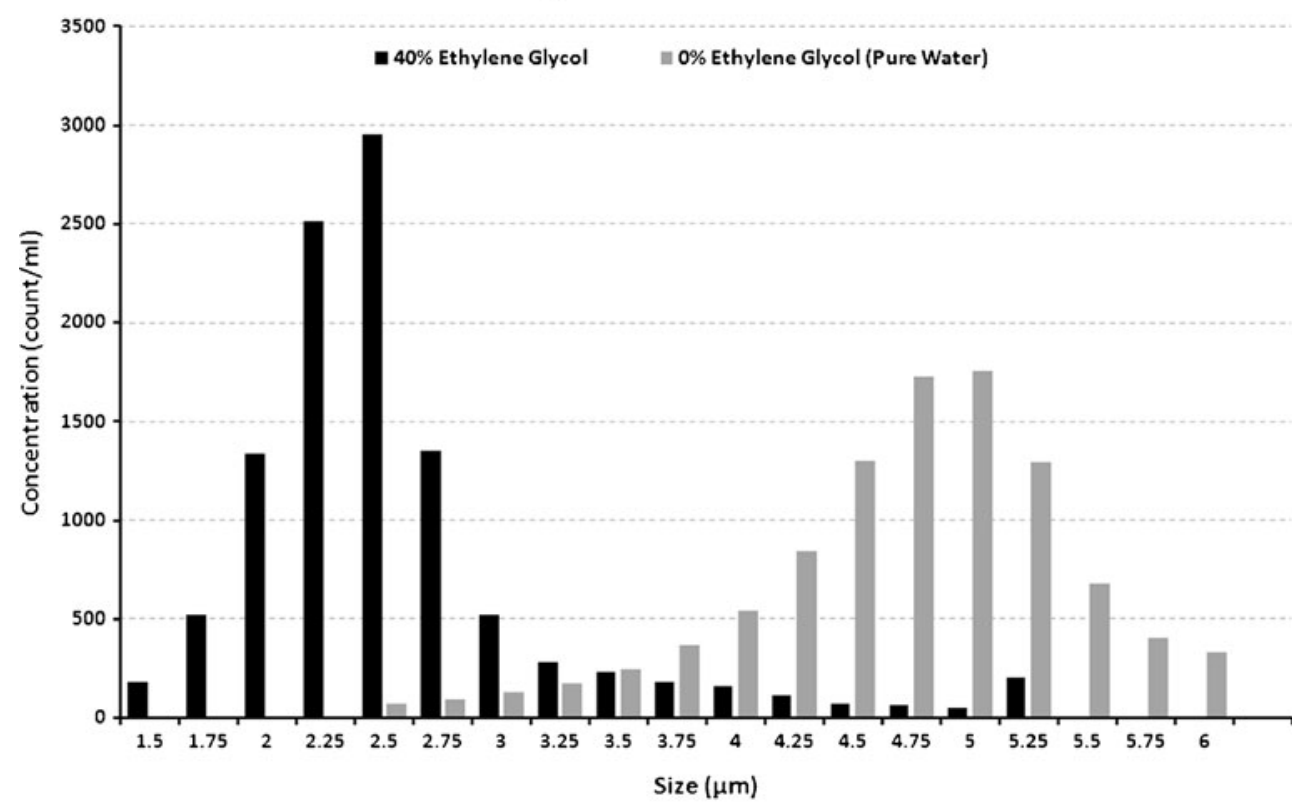

b

Flow Microscopy (MFI)

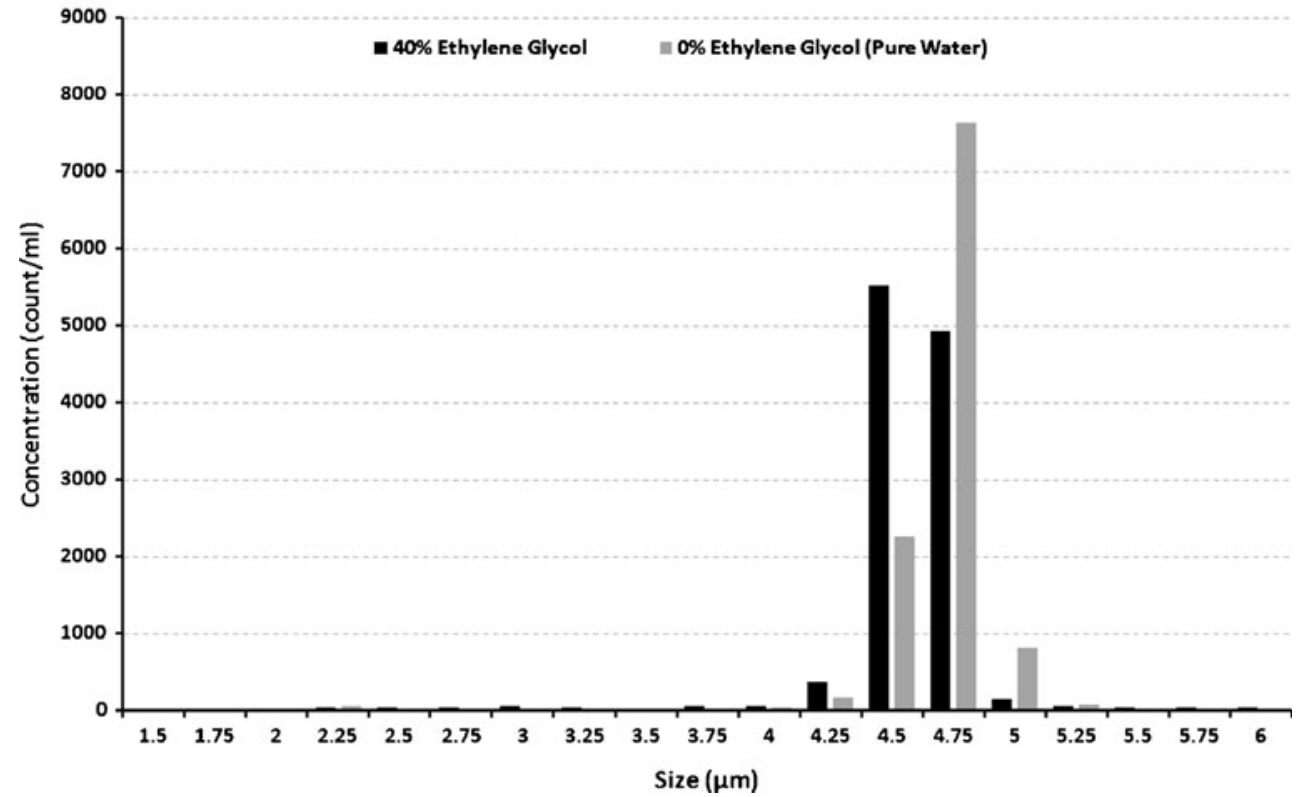

Fig. 2. Measurement of low refractive index glass beads by (a) LO and (b) MFI. Significant under-sizing is seen for the light obscuration measurement in the higher refractive index ethylene glycol mixture $(40 \%, v / v)$ while flow microscopy results were almost unaffected

In a further study of stress-induced aggregates (5), protein particles were induced by shaking a model protein formulation IMGN901 (13) provided by ImmunoGen (Waltham, MA, USA) at a concentration of $1 \mathrm{mg} / \mathrm{mL}$. The number and size distribution of these aggregates were measured by MFI and LO. Differences in the number of particles measured by the two techniques were similar to earlier results. MFI images also showed that the shape of the protein particulates changed during agitation (images not shown).

\section{Evaluation of Filtrate Liquids}

Filtering (normally $0.22 \mu \mathrm{m}$ ) is employed for parenteral products to remove particles in the USP $<788>$ size range. To investigate the effect of filtration on the number and size distribution of protein particles, formulation samples containing agitation stress-induced aggregates were measured pre- and post-filtration $(0.22 \mu \mathrm{m}$ membrane filter, Millipore, USA) by MFI and LO techniques (5). Results again showed that MFI measured more particles (by two orders of magnitude) than LO 

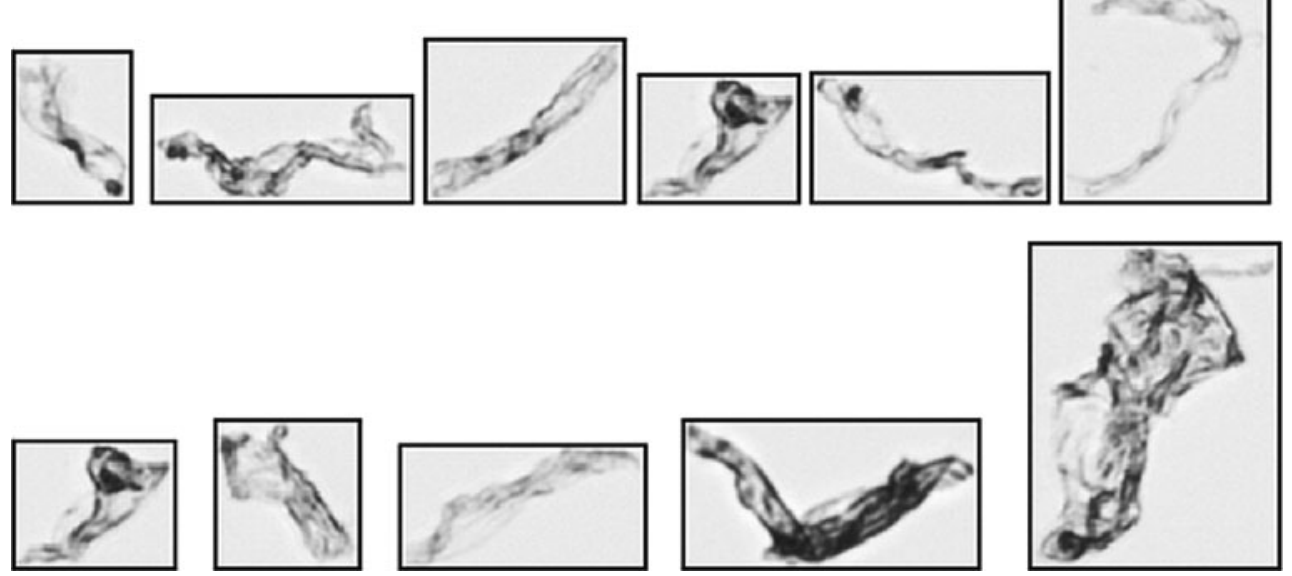

Fig. 3. Representative images of protein particles (size range from 25 to $354 \mu \mathrm{m}$ ) observed by MFI in parenteral samples. The protein particles were observed to be highly heterogeneous in shape, ranging from small dense fibers to large ribbon-like aggregates

with the maximum difference occurring at the smaller size ranges. While it appears that filtration can substantially reduce the concentration of protein particles, particles may still be observed downstream of an intact filter (14,15). It is unknown whether these particles passed through the filter or were generated by the process of filtration. The data also suggest that filtration's effectiveness is dependent on the initial number of sub-visible particles present. This may have implications on strategies to minimize or eliminate administration of protein particles.

\section{High-Concentration Protein Formulations}

In order to develop drugs requiring higher dosages, higher concentrations $(>100 \mathrm{mg} / \mathrm{mL})$ are often employed in protein formulations (16). Properties of such formulations can pose additional challenges to particle measurement techniques. In addition to accelerated aggregation rates at high protein concentrations, high-concentration antibody formulations may exhibit increased opalescence and viscosity (17). Studies have been conducted using MFI on formulations having protein concentrations ranging from 60 to $150 \mathrm{mg} / \mathrm{mL}$ with varying opalescence, viscosity, and carrier fluid refractive index.

\section{Opalescence}

The enhanced opalescence of concentrated monoclonal antibody $(\mathrm{mAb})$ solutions is expected to decrease the contrast between the medium and the translucent proteinaceous particles, potentially making their detection more challenging for any optical measurement. A study was performed to investigate the accuracy of MFI to size and count particles (polystyrene and glass beads) and proteinaceous particles in a concentrated antibody solution $(\sim 60 \mathrm{mg} / \mathrm{mL})$ with a nephelometric turbidity of 15 NTUs (18). Results showed that MFI can detect proteinaceous particles, even in the context of opalescent formulations. The sizing and concentration accuracy of MFI for polystyrene and glass particles, representing different particle refractive index and transparency characteristics, was unaffected by the opalescence of the medium. MFI also demonstrated a high degree of linearity for quantifying proteinaceous particles in opalescent solutions over a wide range of particle concentrations ( $\sim 20$ to 160,000 particles/ $\mathrm{mL}$ ). These particles were significantly underestimated by the LO and manual microscopic technique, particularly those in the $2-10-\mu \mathrm{m}$ size range. The results obtained during this study suggest that, to ensure comprehensive detection of particulates when analyzing opalescent solutions, it is important to use filtered protein solutions instead of buffer for illumination optimization of MFI.

\section{Viscosity}

Higher protein concentration formulations often have higher viscosities (17). To investigate the effect of increasing viscosity, solutions of $10,20,30,40$, and $50 \mathrm{cP}$ were prepared using carboxymethyl cellulose in filtered water. Viscosities were measured using a Gilmont Viscometer (Thermo Fisher Scientific, USA) at room temperature. These solutions were spiked with a nominal concentration of 40,000 particles $/ \mathrm{mL}$ of $10 \mu \mathrm{m}$ polystyrene beads, and the particle size and concentration was measured using MFI. Results showed that the viscosity of the solutions did not impact the calibration of the pump and the volume dispensed. Size measurements were accurate to $\pm 0.02 \mu \mathrm{m}$ for all viscosities. However, reductions in measured concentrations of $10 \%$ to $17 \%$ were observed for higher viscosity samples. Potential contributors to these reductions include sample preparation, particle adhesion, and variations in the flow characteristics of the system. These are currently being investigated.

\section{Carrier Refractive Index}

A further observation in high-concentration protein samples is the increased refractive index of the fluid component relative to any particles which are present. As previously discussed, this could result in a reduction in contrast, causing a reduced efficiency in detecting highly transparent particles. During one such study, a concentrated monoclonal antibody formulation $(150 \mathrm{mg} / \mathrm{mL})$ with a viscosity of $10 \mathrm{cP}$ was used to investigate the accuracy and precision of MFI. The protein formulation was spiked with a known population of silicone oil droplets and serially diluted with the formulating buffer to evaluate the linearity of 
concentration measurement. Non-linear behavior was observed in this particular formulation at higher dilutions. This was initially attributed solely to the higher carrier fluid refractive indices associated with higher protein concentrations. The refractive index of the $150-\mathrm{mg} / \mathrm{mL} \mathrm{mAb}$ formulation decreased progressively from 1.371 to 1.342 on dilution. Subsequent work showed that the dilution process itself rapidly produced additional particulates in this particular formulation. The degree of additional particulate formation also depended greatly on the sample preparation protocol. Further work is underway on instrument configurations and test protocols to understand these effects.

\section{USING MORPHOLOGY FILTERS TO ISOLATE PARTICLE SUB-POPULATIONS}

A sub-population of particles which possesses distinct particle morphology may be isolated using the image filtering capabilities of MFI. Diffraction and pixilation effects limit the minimum particle size where additional useful morphological parameters can be obtained. This limit is approximately $4 \mu \mathrm{m}$ for typically configured instruments as shown in Table I. Morphology-based filtering has been used to separate several of the sub-population types which are often found along with protein particles.

Silicone oil micro-droplets derived from detached lubricants are often present in protein formulations (19). It is desirable that sub-populations of protein aggregates and silicone oil micro-droplets can be independently enumerated when both are present in a sample. MFI has been successfully used to accomplish this with an accuracy of $\sim 96 \%$ for particles $\geq 5 \mu \mathrm{m}$ employing a simple aspect ratio filter $(3,4)$.

Air/gas bubbles can be also be present as a result of handling processes, particularly in the case of reconstituted lyophilized products. When measuring populations of solid particles suspended in these fluids, it is desirable that bubbles can be identified and removed from the statistics. A drug formulation sample which had exhibited high particle counts $\geq 10 \mu \mathrm{m}$ when measured by LO was tested by MFI (3). A circularity filter was first applied to the MFI data to identify single air bubbles. Remaining bubble clusters (coalescent air bubbles) were identified using an intensity/aspect ratio filter.

An example of a filtering application for multiple subpopulations is shown in Fig. 4. The results show that the use of a minimum intensity filter provides good separation between protein particles, air bubbles, and silicone oil micro-droplets suspended in PBS solutions. This separation can be further improved by the use of a multi-parameter filter. When multiple sub-populations and more complex objects are present, MFI software provides a systematic means of developing, testing, and sequentially applying more multi-stage filters.

\section{REPEATABILITY AND CONSISTENCY OF MFI MEASUREMENT}

The capabilities of MFI technology for measuring protein formulations has already resulted in MFI being widely employed for sub-visible particulate analysis during formulation development. However, in order to be recognized as a viable candidate for regulatory data collection and quality assurance, MFI assays must be shown to provide consistent and accurate measurements for the particle populations found in the drug to which the assays will be applied. Validation methods must ensure that measurements will be consistent and repeatable for multiple instruments for the manufacturing life of a formulation and must provide accurate product monitoring with sensitive detection for outof-limit variations while avoiding unnecessary lot rejections

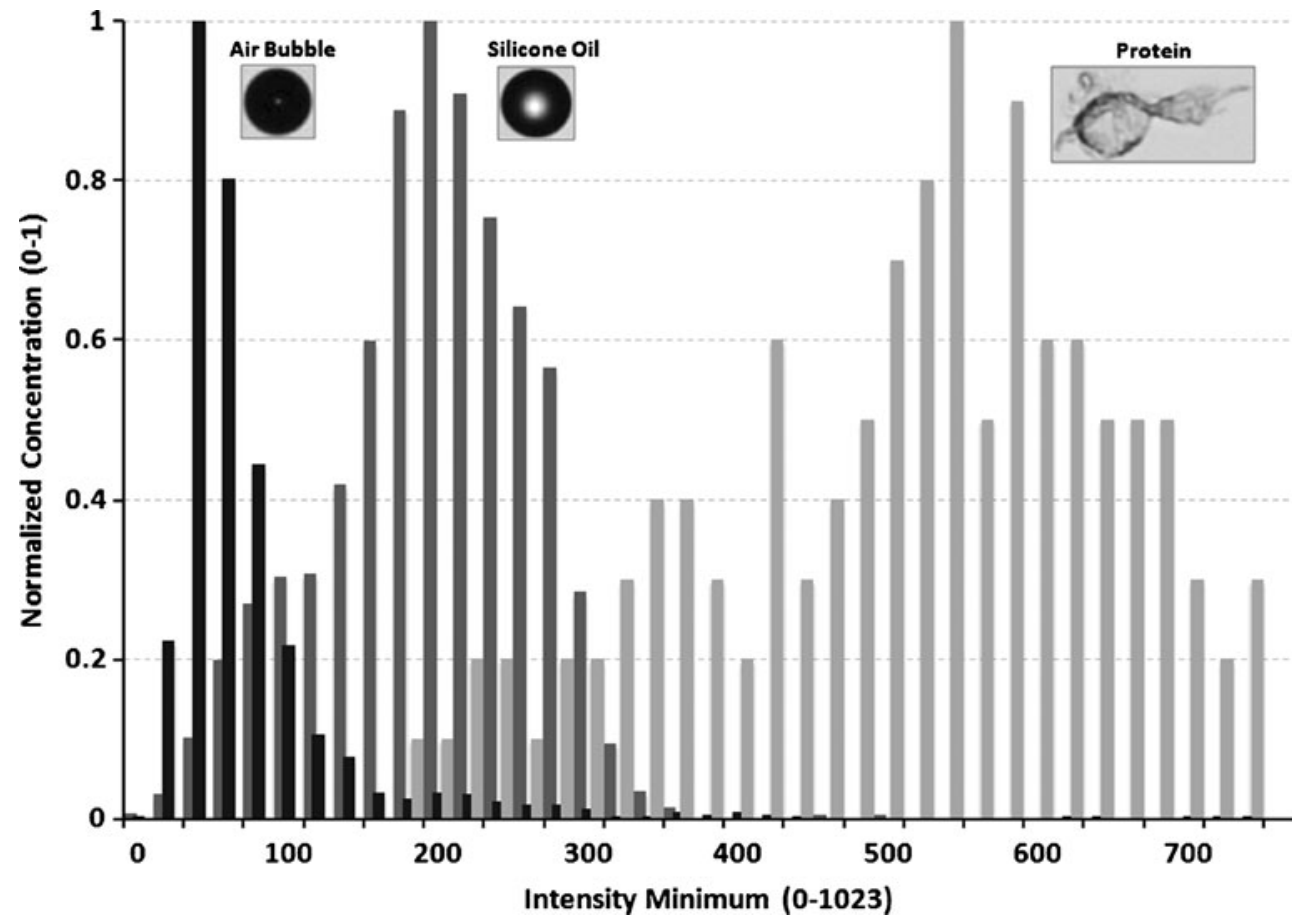

Fig. 4. Image filtering capabilities of MFI. An intensity minimum filter was applied to a mixed particle population to distinguish protein aggregates, silicone oil droplets, and air bubbles 
which might result from test method inaccuracies. While MFI has been successfully applied to a range of drug types, the specific properties of each new protein drug and its associated particle population may influence the optimum protocol for MFI testing, data analysis, and reporting. MFI methods should be optimized and validated for each drug on which they are to be applied. Properties which will influence MFI testing protocol include the fragility and time stability of particle populations, statistical accuracy requirements, settling properties, turbidity, viscosity, and the presence of extrinsic particles.

To illustrate the repeatability and consistency which should reasonably be expected in routine testing for a protein-based drug, intra-instrument and inter-instrument variations were measured for a model protein drug formulation (IgG1) at a concentration of $1 \mathrm{mg} / \mathrm{mL}$. Sub-visible protein aggregates were created in the $\mathrm{IgG} 1 \mathrm{mAb}$ solution using a freeze-thaw method. The freeze-thaw method consisted of repeatedly freezing the protein sample at $-80^{\circ} \mathrm{C}$ for $5 \mathrm{~min}$ and immediately thawing in a warm water bath at $37^{\circ} \mathrm{C}$ for $5 \mathrm{~min}$. The freeze-thaw cycle was performed five times above and beyond the original stock sample thaw. The thawed sample was inverted several times to ensure proper mixing and diluted 10-fold with filtered placebo buffer (PBS, $\mathrm{pH} 7.0 \pm 0.2$ ). The count and size of the protein particulates were analyzed using MFI. Samples were introduced using pipette tips. Prior to each sample run, particle-free fluid was flushed through the system to provide a clean baseline. For each sample and control, four separate runs of $1 \mathrm{~mL}$ each were analyzed where the first run was discarded and the remaining three were averaged. Appropriate negative controls were measured to ascertain the influence of the placebo buffer. Three standard DPA 4200 instruments validated with polystyrene beads and a sensitivity challenge standard were employed during this study. Intra- and inter-instrument variations in the total particle population were measured by the three instruments as a function of the age of the protein sample. Higher intra-instrument variability ( RSD $_{\text {Intra }} 15 \%$ ) was observed in the initial particle count at $0 \mathrm{~h}$ (Fig. 5a), indicating that particle population was initially unstable following dilution with PBS. This is an example of the type of instability which can invalidate dilution series measurements on protein particles. The intra-instrument variability decreased $\left(\mathrm{RSD}_{\text {Intra }} 4 \%\right)$, after $1 \mathrm{~h}$ of incubation at room temperature, demonstrating that the particle population stabilized over time. It is hypothesized that, although the protein particles were diluted with placebo buffer and there is no change in solution conditions (except for a reduction in protein concentration), the protein population appears to be in an unstable state after initial dilution and requires time to stabilize. This observation needs further investigation. Interinstrument variability was found to be small for all time points measured compared to the intra-instrument variability. Inter- and intra-instrument variations in the total particle population also depended on particle size (Fig. 5b), as might be expected from statistical factors, where higher variability was seen in higher particle size ranges where fewer particles were present. Results demonstrate that, for this typical aggregate population, MFI measurements using appropriately validated instruments and an optimized test protocol can provide high repeatability and accuracy.

\section{TECHNOLOGY OPTIMIZATION}

As a result of protein particle studies, a number of areas were identified where the MFI technology could be optimized to better meet the requirements of this important but challenging application. These include:

- Maximizing and controlling instrument sensitivity for highly transparent particles

- Achieving high sampling efficiency for low sample volume

- Minimizing shear stress on the sample

- Reducing the minimum volume of sample required for a measurement

\section{Maximizing and Controlling Instrument Sensitivity}

Measurements on the same sample by different instruments may vary depending on small variations in instrument sensitivity (i.e., translucent particle detection). These effects may be reduced by employing a fixed threshold having the minimum value consistent with optical noise. It was further found that the range of optical adjustment where accurate measurements of polystyrene beads can be obtained still allows for significant variations in the measurement of more challenging protein particle populations. This was attributed to the fact that polystyrene bead standards, unlike protein particles, are easily detected and do not challenge the sensitivity of the optical particle analyzer. A further sensitivity challenge standard was required which would allow different instruments to be assessed and adjusted to achieve consistent results when very low contrast particles were present. Protein particle populations themselves are not sufficiently stable or repeatable for this purpose, especially when longer-term and multi-site comparisons are required. To address this issue, a stable, mineral-based suspension was identified which had similar optical properties and measurement behavior as a challenging protein aggregate population. Individual instruments were then optically configured and verified for achieving consistent particle distributions using this surrogate. This approach was found to provide high sensitivity and minimize inter-instrument differences. While general purpose MFI instruments can possess a number of magnification settings for use with flow cells of different depths, research with protein aggregate formulations demonstrated that this flexibility is not consistent with the fine adjustments required to obtain acceptable repeatability between instruments. Single purpose, optimized configurations covering the size range of interest (i.e., 1 to $70 \mu \mathrm{m}$ or 2 to $300 \mu \mathrm{m}$ ) could provide more consistent results.

\section{Maximizing Sampling Efficiency}

The sampling efficiency in MFI is the proportion of the total fluid volume passing through the flow cell compared to the fluid which is captured in image frames. Because small quantities of the sample fluid lie outside the field of view or pass between successive frames, not all the sample which passes through the flow cell is analyzed, and thus, the sampling efficiency is not $100 \%$. When analyzing small volumes, investigating rare events, and adapting to existing sampling schemes, it is desirable that sampling efficiency be well quantified and as 

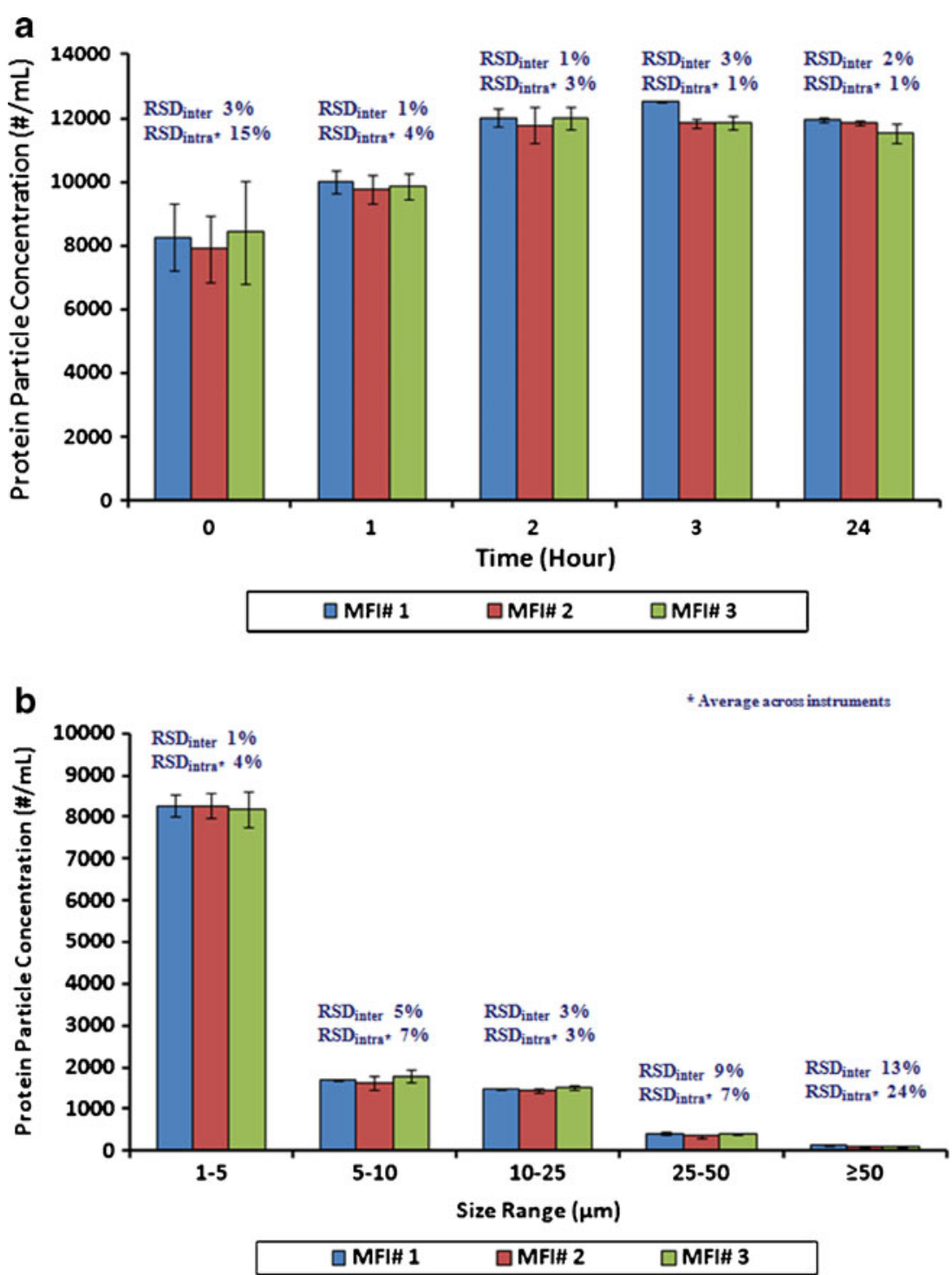

Fig. 5. Inter- and intra-MFI instrument repeatability (a); IgG1 particle sample reproducibility in various size ranges (b). Results demonstrate that, for this typical aggregate population, MFI measurements using appropriately validated instruments and an optimized test protocol can provide high repeatability and accuracy

high as possible without particles appearing in successive frames. By narrowing the flow cell channel and arranging that successive frames lie as close together as possible without overlap, a sampling efficiency was achieved which exceeded $85 \%$. The combination of high sampling efficiency and sensitivity allows samples with only a few particles per milliliter to be detected and accurately measured.

\section{Minimizing Shear Forces}

When delicate particles are subjected to shear forces resulting from stirring or rapid sample flow, particles may fragment before they are measured. In protein-based drug formulations, shear stresses can also result in the rapid formation and growth of aggregate particles. The MFI sample introduction system was modified to minimize shear forces by using a gravity-assisted design to ensure large and dense particles enter the viewing zone without the need for high flow rates. When stirring was required to maintain particles in suspension, it was accomplished using gentle, adjustable, and precise stirring system.

\section{Minimizing Sample Volume Requirement}

The volume of material available for particle analysis is often very limited, especially in early stage formulation development. By reducing the volume of fluid in the sample application lines and the flow cell, flushing requirements to achieve clean baselines and minimum sample volumes are reduced. A modified sample application port was developed which directly accepts pipette tips or standard Luer connections to larger sample containers. This facilitates obtaining 
clean baselines and allows both smaller volume $(<0.5 \mathrm{~mL})$ and larger volume samples to be conveniently introduced.

\section{DISCUSSION}

Currently, there are no regulatory limits for sub-visible particles smaller than $10 \mu \mathrm{m}$. In a recently published commentary, it was noted that there are critical gaps in the detection and control of sub-visible proteinaceous particles, especially in the size range $0.1-10 \mu \mathrm{m}$ and that these particles may have immunogenic potential (1). In this review, we have described the capability of MFI to measure proteinaceous particle populations. Results show that the MFI technique provides high sensitivity in measuring such particles, the capability to independently analyze sub-populations, and yields additional insights provided by particle images.

Measurements made by MFI have been compared with those obtained by LO and membrane microscopy. For the range of sample types evaluated, these standard techniques were found to detect a small proportion of the particles present, and this may not be adequately representative of the total sub-visible population. The lack of sensitivity in LO in detecting transparent protein particles in carrier fluids of similar refractive index has been shown to be in part due to the technique's calibration method. Assessing the absolute accuracy of any method in this application is compounded by the absence of consensus protein particle standards.

Work on a variety of protein formulations has shown that a number of sample-specific factors including opacity, viscosity, and particle transparency should be considered in designing and validating an MFI-based measurement protocol for a particular drug. One notable observation in some formulations is the potential for rapid formation of additional particles following dilution. This effect can preclude the use of dilution series with these formulations as an element of method validation.

Areas of optimization for MFI technology to better meet the requirements for protein particle measurement have been described. In the course of investigating the validation of the technology for quality assurance applications, intra- and interinstrument variability studies have been performed for optimized instruments using a model aggregate population and model measurement protocol. These have demonstrated the potential for obtaining highly consistent results from the technology.

\section{CONCLUSION}

Flow microscopy is an emerging technology which, when suitably optimized, can provide a number of advantages over existing techniques in the analysis of sub-visible particles in protein formulations. These include:

- Improved sensitivity and accuracy

- Measurements which are less dependent on particle composition

- Minimal sample stress

- Software-based identification and filtering of particle sub-populations
The advantages of MFI are proving to be of value in a range of tasks in formulation research and development (26). Further experience and refinement in the technology may result in its use as an accepted method for performing measurements on sub-visible particles in protein formulations. Such measurements could assist in minimizing protein aggregation and in maintaining the consistency in the size distribution and morphology of residual particle populations during formulation development, clinical trials, manufacturing, and storage.

\section{ACKNOWLEDGMENTS}

The authors wish to thank ImmunoGen, Eli Lilly \& Co., and Genentech for providing permission to release data used in this manuscript.

\section{REFERENCES}

1. Carpenter J, Randolph TW, Jiskoot W, Crommelin D, Middaugh C, Winter G, et al. Overlooking sub-visible particles in therapeutic protein products: gaps that may compromise product quality. J Pharm Sci. 2008;98(4):1201-5.

2. Ives C, Soderquist R, Stoner M, Kendrick B. Light obscuration particulate analysis for protein solutions: challenges and limitations. Protein Stability Conference. 2007 Breckenridge (CO), USA.

3. Sharma D, King D, Moore P, Oma P, Thomas D. Flow microscopy for particulate analysis in parenteral and pharmaceutical fluids. Eur J Parent Pharm Sci. 2007;12(4):97-101.

4. Sharma D, Oma P, Krishnan S. Silicone micro-droplets in protein formulations-detection and enumeration. Pharma Tech. 2009;33(4):74.

5. Huang C, Sharma D, Oma P, Krishnamurthy R. Quantitation of protein particles in parenteral solutions using micro-flow imaging. J Pharm Sci. 2009;98(9):3058-71.

6. Huang C, Sharma D, Amplett G, Oma P, Krishnamurthy K. Quantitation of protein particles in parenteral solutions using light obscuration and micro-flow imaging. Protein Stability Conference. 2007 Breckenridge (CO), USA.

7. Wen J, Arakawa T. Refractive index of proteins in aqueous sodium chloride. Anal Biochem. 2000;280:327-9.

8. Ball V, Ramsden JJ. Buffer dependence of refractive index increments of protein solutions. Biopolym. 1998;46:489-92.

9. Chi EY, Krishnan S, Randolph TW, Carpenter JF. Physical stability of proteins in aqueous solution: mechanism and driving forces in non-native protein aggregation. Pharm Res. 2003;20 (9):1325-36.

10. Chi EY, Weickmann J, Carpenter JF, Manning MC, Randolph TW. Heterogeneous nucleation-controlled particulate formation of recombinant human platelet-activating factor acetylhydrolase in pharmaceutical formulation. J Pharm Sci. 2005; 94(2):256-74.

11. Sluzky V, Tamada JA, Klibanov AM, Langer R. Kinetics of insulin aggregation in aqueous solutions upon agitation in the presence of hydrophobic surfaces. Proc Natl Acad Sci USA. 1991;88(21):9377-81.

12. Mahler HC, Muller R, Friess W, Delille A, Matheus S. Induction and analysis of aggregates in a liquid IgG1-antibody formulation. Eur J Pharm Biopharm. 2005;59(3):407-17.

13. ImmunoGen. http://www.immunogen.com/wt/page/IMGN901b. Accessed $20 \mathrm{Jul} 2008$.

14. Groves MJ. Particulate contamination in parenterals: current issues. Boll Chim Farm. 1991;130(9):347-54.

15. Ball PA, Bethune K, Fox J, Ledger R, Barnett M. Particulate contamination in parenteral nutrition solutions: still a cause for concern? Nutrition. 2001;17(11-12):926-9. 
16. Shire SJ, Shahrokh Z, Liu J. Challenges in the development of high protein concentration formulations. J Pharm Sci. 2004;93 (6):1390-402.

17. Liu J, Nquyen MDH, Andya JD, Shire SJ. Reversible selfassociation increases the viscosity of a concentrated monoclonal antibody in aqueous solution. J Pharm Sci. 2005;94:1928-40.
18. Sharma D, Oma P, Pollo MJ, Sukumar M. Quantification and characterization of subvisible proteinaceous particles in opalescent $\mathrm{mAb}$ formulations using micro-flow imaging. J Pharm Sci. 2009;99:2628-42. doi:10.1002/jps.22046.

19. Jones L, Kaufmann A, Middaugh C. Silicone oil induced aggregation of proteins. J Pharm Sci. 2005;94:918-27. 